\title{
Some properties of lattice OWA operators and their importance in image processing
}

\author{
G. Ochoa ${ }^{1}$ I. Lizasoain ${ }^{1}$ D. Paternain ${ }^{2}$ H. Bustince ${ }^{2}$ N.R. Pal $^{3}$ \\ ${ }^{1}$ Dpto. Matemáticas, Univ. Pública de Navarra, Campus Arrosadía, 31006 Pamplona, Spain \\ ${ }^{2}$ Dpto. Automática y Computación, Univ. Pública de Navarra, Campus Arrosadía, 31006 Pamplona, Spain \\ ${ }^{3}$ Dep. Electronic and Communication Sciences Unit, Indian Statistical Institute, 700108 Calcutta, India
}

\begin{abstract}
In this work we deal with the problem of using OWA operators in color image reduction algorithms. For this reason, we study OWA operators defined on an arbitrary finite lattice endowed with a t-norm and a t-conorm. In the case of RGB color images, we apply OWA operators defined on a finite product lattice. Depending on the OWA operator considered, we see that the reduced images become brighter or darker. Then, we make a preliminary study of a qualitative orness measure for classifying each lattice OWA operator according to its proximity to the maximum of the given data.
\end{abstract}

Keywords: OWA operator, lattice-valued fuzzy sets, orness, t-norm, t-conorm, image reduction.

\section{Introduction}

Aggregation functions are a very important tool in problems of data fusion. In particular, in image processing it is very usual to aggregate or fuse several inputs into a representative output, as in image filtering, stereo vision, segmentation, among others. In this work we focus on image reduction algorithms. These algorithms reduce the dimension of an image while keeping as much information as possible. From an applied point of view, image reduction can be used to accelerate computation on an image or to reduce its storage or transmission cost.

When we deal with grayscale images, each pixel is associated with its intensity value, typically an integer between 0 (black) and 255 (white). The intensity of a pixel can be easily transformed into a real number in the interval $[0,1]$ just by dividing by 255 . In order to reduce the spatial resolution of the image, in [7] the use of averaging aggregation functions has been widely studied. The authors propose to aggregate non-overlapping blocks of pixels into a single one. Evidently, the quality of the reduced image directly depends on the aggregation function considered.

One of the most versatile functions used in image reduction are OWA operators [8]. Each OWA operator provides a weighted average of the intensities within a block, where each weight associated to each intensity only depends on the their order.
Having this in mind, we can for example prioritize high intensities so that the resulted image becomes very bright. Or, on the contrary, we can prioritize low intensities to obtain darker reduced images. In order to classify each OWA operator attending to their behaviour, in [9] proposed the orness of an OWA operator as a measure of the proximity to an OR aggregation.

However, when we deal with color images, the use of aggregation functions is not trivial. In the RGB color scheme, for example, each pixel is associated with three different intensities, corresponding to red, green and blue colors. In [1], the authors propose the use of aggregation functions defined on a Cartesian product of lattices in order to reduce images in the RGB color scheme. Following these ideas, in this work we propose the study of lattice OWA operators for reducing color images. As in the case of OWA operators (defined on $[0,1]$ ) we are interested in classifying each operator attending to its behaviour. For this reason, we start studying a qualitative orness measure for lattice OWA operators. This measure will give us the information of the behaviour of the operator in each color channel.

The paper is organized as follows. Section 2 is devoted to revisit results concerning to t-norms, t-conorms and aggregation functions defined on bounded lattices, especially OWA operators. Section 3 introduces and analyzes the concept of qualitative orness for OWA operators defined on finite lattices. Section 4 is devoted to OWA operators defined on product lattices. In Section 5 we analyze the effect of OWA operators in color image reduction. A final section of conclusions closes the paper.

\section{Preliminaries}

\subsection{OWA operators}

In [8], an $n$-ary ordered weighted average (OWA) operator is defined for values in $[0,1]$ in the following way.

Definition 1 (Yager [8]). Let $\alpha=\left(\alpha_{1}, \cdots, \alpha_{n}\right) \in$ $[0,1]^{n}$ be a weighting vector with $\alpha_{1}+\cdots+\alpha_{n}=1$. An $n$-ary OWA operator is a map $F_{\alpha}:[0,1]^{n} \rightarrow$ $[0,1]$ given by

$$
F_{\alpha}\left(a_{1}, \cdots, a_{n}\right)=\alpha_{1} b_{1}+\cdots+\alpha_{n} b_{n},
$$


where $\left(b_{1}, \ldots, b_{n}\right)$ is a rearrangement of $\left(a_{1}, \cdots, a_{n}\right)$ satisfying $b_{1} \geq \cdots \geq b_{n}$.

In [9] Yager introduces an orness measure for each OWA operator, which depends only on the weighting vector $\alpha=\left(\alpha_{1}, \ldots, \alpha_{n}\right)$.

$$
\operatorname{orness}\left(F_{\alpha}\right)=\frac{1}{n-1} \sum_{i=1}^{n}(n-i) \alpha_{i}
$$

Notice that the orness of the OR-operator is equal to 1 , while the orness of the AND-operator is equal to 0 . Moreover, if $\alpha_{1}=\cdots=\alpha_{n}=\frac{1}{n}$, then $\operatorname{orness}\left(F_{\alpha}\right)=0.5$.

\subsection{Lattice OWA operators}

In order to define lattice OWA operators, we start by recalling some concepts [3].

Throughout this paper $\left(L, \leq_{L}\right)$ will denote a bounded lattice, i.e., a partially ordered set in which every two elements have both a supremum and an infimum and there are two elements, $1_{L}$ and $0_{L}$, that are respectively the greatest and the least elements of $L$. In addition, $\left(L, \leq_{L}, T, S\right)$ will denote a bounded lattice endowed with a t-norm $T$ and a t-conorm $S$.

Definition 2 (see [2]). A map $T: L \times L \rightarrow L$ is said to be a t-norm [resp. t-conorm] on $\left(L, \leq_{L}\right)$ if it is commutative, associative, increasing in each component and has a neutral element $1_{L}$ [resp. $\left.0_{L}\right]$.

Definition 3 (see [4]). Let $\left(L, \leq_{L}\right)$ be a bounded lattice. An $n$-ary aggregation function is a function $M: L^{n} \rightarrow L$ such that:

1. $M\left(a_{1}, \ldots, a_{n}\right) \leq_{L} \quad M\left(a_{1}^{\prime}, \ldots, a_{n}^{\prime}\right)$ whenever $a_{i} \leq_{L} a_{i}^{\prime}$ for $1 \leq i \leq n$.

2. $M\left(0_{L}, \ldots, 0_{L}\right)=0_{L}$ and $M\left(1_{L}, \ldots, 1_{L}\right)=1_{L}$.

An $n$-ary aggregation function $M$ is said to be idempotent if $M(a, \ldots, a)=a$ for every $a \in L$. It is said to be symmetric if, for every permutation $\sigma$ of the set $\{1, \ldots, n\}, M\left(a_{1}, \ldots, a_{n}\right)=$ $M\left(a_{\sigma(1)}, \ldots, a_{\sigma(n)}\right)$.

In [5] $n$-ary ordered weighted average (OWA) operators are extended to any bounded lattice endowed with a t-norm $T$ and a t-conorm $S$ whenever the weighting vector satisfies some distributivity condition. Before reminding this extension, we bring together some necessary definitions.

Definition 4 ([5]). Consider any bounded lattice $\left(L, \leq_{L}, T, S\right) . \alpha=\left(\alpha_{1}, \ldots, \alpha_{n}\right) \in L^{n}$ is said to be a weighting vector in $\left(L, \leq_{L}, T, S\right)$ if $S\left(\alpha_{1}, \ldots, \alpha_{n}\right)=$ $1_{L}$ and it is called a distributive weighting vector in $\left(L, \leq_{L}, T, S\right)$ if it also satisfies that $T\left(a, S\left(\alpha_{1}, \ldots, \alpha_{n}\right)\right)=S\left(T\left(a, \alpha_{1}\right), \ldots, T\left(a, \alpha_{n}\right)\right)$ for any $a \in L$.
Remark 5. If $L$ is the real interval $[0,1]$ with the usual order $\leq, T(a, b)=a b$ for every $a, b \in[0,1]$ and $S(a, b)=\min \{a+b, 1\}$ for every $a, b \in[0,1]$, then $\alpha=\left(\alpha_{1}, \ldots, \alpha_{n}\right) \in[0,1]^{n}$ with $S\left(\alpha_{1}, \ldots, \alpha_{n}\right)=1$ is a distributive weighting vector if and only if $\alpha_{1}+\cdots+\alpha_{n}=1$, where $S\left(\alpha_{1}, \ldots, \alpha_{n}\right)$ means $S\left[\ldots\left(S\left(S\left(\alpha_{1}, \alpha_{2}\right), \alpha_{3}\right), \ldots \alpha_{n-1}\right), \alpha_{n}\right]$.

Definition $6([5])$. Let $\left(L, \leq_{L}, T, S\right)$ be a complete lattice. For any vector $\left(a_{1}, \ldots, a_{n}\right) \in L^{n}$, a totally ordered vector $\left(b_{1}, \ldots, b_{n}\right)$ is defined by

$$
\begin{aligned}
& \text { - } b_{1}=a_{1} \vee \cdots \vee a_{n} \in L . \\
& \text { - } b_{2}=\left[\left(a_{1} \wedge a_{2}\right) \vee \cdots \vee\left(a_{1} \wedge a_{n}\right)\right] \vee\left[\left(a_{2} \wedge a_{3}\right) \vee\right. \\
& \\
& \left.\cdots \vee\left(a_{2} \wedge a_{n}\right)\right] \vee \cdots \vee\left[a_{n-1} \wedge a_{n}\right] \in L . \\
& \quad \vdots \\
& \text { - } b_{k}=\vee\left\{a_{j_{1}} \wedge \cdots \wedge a_{j_{k}} \mid j_{1}<\cdots<j_{k} \in\right. \\
& \quad\{1, \ldots, n\}\} \in L . \\
& \quad \vdots \\
& \text { - } b_{n}=a_{1} \wedge \cdots \wedge a_{n} \in L .
\end{aligned}
$$

Remark 7. Let $\left(L, \leq_{L}, T, S\right)$ be a bounded lattice, $\left(a_{1}, \ldots, a_{n}\right) \in L^{n}$ and $\left(b_{1}, \ldots, b_{n}\right)$ as defined above.

1. $a_{1} \wedge \cdots \wedge a_{n}=b_{n} \leq_{L} b_{n-1} \leq \cdots \leq_{L} b_{2} \leq_{L} b_{1}=$ $a_{1} \vee \cdots \vee a_{n}$.

2. If $n$ is odd and $k=\frac{n+1}{2}$, then $b_{k}$ agrees with the $n$-ary median function of $\left(a_{1}, \ldots, a_{n}\right)$.

3. If the set $\left\{a_{1}, \ldots, a_{n}\right\}$ is totally ordered, then $\left(b_{1}, \ldots, b_{n}\right)$ is a rearrangement of it. In fact, each $b_{k}$ is the $k$-th statistic in this case.

Definition 8 ([5]). Let $\left(L, \leq_{L}, T, S\right)$ be a bounded lattice. For each distributive weighting vector $\alpha=\left(\alpha_{1}, \ldots, \alpha_{n}\right) \in L^{n}$, the function $F_{\alpha}$ : $L^{n} \rightarrow L$ given for any $\left(a_{1}, \ldots, a_{n}\right) \in L^{n}$ by $F_{\alpha}\left(a_{1}, \ldots, a_{n}\right)=S\left(T\left(\alpha_{1}, b_{1}\right), \cdots, T\left(\alpha_{n}, b_{n}\right)\right)$, is called an $n$-ary $O W A$ operator.

(The vector $\left(b_{1}, \ldots, b_{n}\right)$ is that defined in Def. 6 for each $\left.\left(a_{1}, \ldots, a_{n}\right) \in L^{n}\right)$.

It is easy to check that $F_{\alpha}$ is a symmetric and idempotent $n$-ary aggregation function which lies between the AND operator, obtained for the weighting vector $\left(0_{L}, \ldots, 0_{L}, 1_{L}\right)$ and the OR operator, got when $\alpha=\left(1_{L}, 0_{L}, \ldots, 0_{L}\right)$.

Moreover, if $\left(L, \leq_{L}, T, S\right)$ is the bounded lattice described in Remark 5 , then for each distributive weighting vector $\alpha=\left(\alpha_{1}, \ldots, \alpha_{n}\right) \in[0,1]^{n}, F_{\alpha}$ agrees with the OWA operator given by Yager.

\section{Qualitative orness measure for OWA operators on finite lattices}

In the case of a finite lattice $\left(L, \leq_{L}, T, S\right)$, we introduce the following definition of a qualitative measure of the orness of an $n$-ary OWA operator, in which, if $L=\left\{a_{1}, \ldots, a_{l}\right\}$, the vector $\left(1_{L}, b_{1}, \ldots, b_{l}\right)$ will play the role of the real $n$-ary vector $\left(0, \frac{1}{n-1}, \frac{2}{n-1}, \cdots, \frac{n-2}{n-1}, \frac{n-1}{n-1}\right)$ in formula (1) for the case that $n-1=l$. Some fitting is necessary in the general case. 
Definition 9. Let $\left(L, \leq_{L}, T, S\right)$ be a finite lattice with $L=\left\{a_{1}, \ldots, a_{l}\right\}$ and call $\left(b_{1}, \ldots, b_{l}\right)$ the vector defined in Def. 6. For any distributive weighting vector $\alpha=\left(\alpha_{1}, \ldots, \alpha_{n}\right) \in L^{n}$, we define a qualitative orness measure of the corresponding OWA operator $F_{\alpha}$ in the following way:

1. Call $m=l(n-1)$.

2. Consider the descending chain $c_{1} \geq_{L} \cdots \geq_{L} c_{m}$ defined by

$c_{1}=\cdots=c_{n-1}=b_{1} ; c_{n}=\cdots=c_{2(n-1)}=$ $b_{2} ; \ldots ; c_{(l-1)(n-1)+1}=\cdots=c_{l(n-1)}=b_{l}$.

Notice that $c_{1}=b_{1}=1_{L}$ and $c_{l(n-1)}=b_{l}=0_{L}$

3. Build a descending subchain of $\left\{c_{1}, \ldots, c_{m}\right\}$ by means of

$d_{1}=1_{L}, d_{2}=c_{l}, d_{3}=c_{2 l}, . ., d_{n}=c_{(n-1) l}=0_{L}$,

i. e. , for each $j \in\{1, \ldots, n-1\}, d_{j+1}=c_{j l}=$ $b_{k}$ with $k=1+\left[\frac{j l-1}{n-1}\right]$, where the symbol $\left[\frac{a}{b}\right]$ denotes the whole part of $\frac{a}{b}$.

4. Call orness $\left(F_{\alpha}\right)=S\left(T\left(\alpha_{1}, d_{1}\right), \ldots, T\left(\alpha_{n}, d_{n}\right)\right)$.

Remark 10. Notice that the definition of $\operatorname{orness}\left(F_{\alpha}\right)$ depends only on the vector $\alpha=$ $\left(\alpha_{1}, \ldots, \alpha_{n}\right)$. So, it is necessary to check that, if $\beta=\left(\beta_{1}, \ldots, \beta_{n}\right)$ is another distributive weighting vector with $F_{\alpha}=F_{\beta}$, then $\operatorname{orness}\left(F_{\alpha}\right)=$ $\operatorname{orness}\left(F_{\beta}\right)$. Indeed, notice that $\operatorname{orness}\left(F_{\alpha}\right)=$ $F_{\alpha}\left(d_{1}, \ldots, d_{n}\right)$.Therefore, if $F_{\alpha}=F_{\beta}$, then $\operatorname{orness}\left(F_{\alpha}\right)=\operatorname{orness}\left(F_{\beta}\right)$ and this concept is welldefined.

In a complementary way, notice that the same chain $d_{1} \geq_{L} \cdots \geq_{L} d_{n}$ is obtained if we let $m$ be any common multiple of $\{l, n-1\}$. In that case, if $m=$ le with $e \in \mathbb{N}$, the chain $c_{1} \geq_{L} \cdots \geq_{L} c_{m}$ must be

$c_{1}=\cdots=c_{e}=b_{1} ; c_{e+1}=\cdots=c_{2 e}=b_{2} ; \ldots ;$ $c_{(l-1) e+1}=\cdots=c_{l e}=b_{l}$ and, if $m=(n-1) h$ with $h \in \mathbb{N}$, then the chain $d_{1} \geq_{L} \cdots \geq_{L} d_{n}$ must be

$d_{1}=1_{L}, d_{2}=c_{h}, d_{3}=c_{2 h}, \ldots, d_{n}=c_{(n-1) h}=0_{L}$.

Then the chain $d_{1} \geq_{L} \cdots \geq_{L} d_{n}$ obtained in this way is the same as that obtained in Def. 9. Indeed, for each $j \in\{1, \ldots, n-1\}, d_{j+1}=c_{j h}=b_{k}$ with $k=1+\left[\frac{j h-1}{e}\right]=1+\left[\frac{j l-1}{n-1}\right]$.

Example 11. Consider the lattice $L=C_{3} \times C_{2}$, where $C_{3}$ and $C_{2}$ are the chains consisting of 3 and
2 elements respectively:

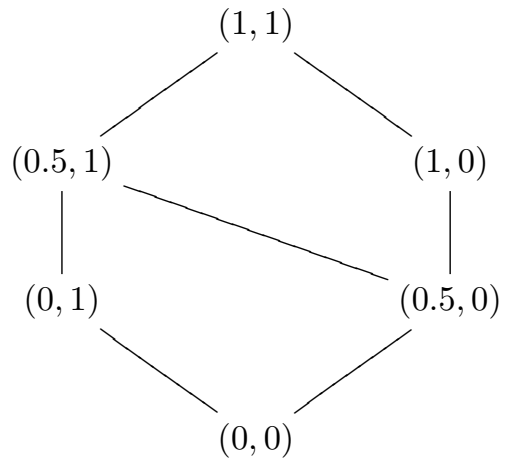

We consider the t-norm given by the meet and the t-conorm given by the join and the OWA operator $F_{\alpha}$ obtained for the weighting vector $\alpha=$ $((0.5,1),(0,1),(0,1),(1,0),(0.5,0)) \in L^{5}$. In order to calculate its orness, write $m=\operatorname{lcm}(|L|, n-$ 1) $=12$.

Consider the descending chain $c_{1} \geq_{L} \cdots \geq_{L} c_{12}$ given by $c_{1}=c_{2}=b_{1}=(1,1) ; c_{3}=c_{4}=b_{2}=$ $(1,1) ; c_{5}=c_{6}=b_{3}=(0.5,1) ; c_{7}=c_{8}=b_{4}=$ $(0.5,0) ; c_{9}=c_{10}=b_{5}=(0,0) ; c_{11}=c_{12}=b_{6}=$ $(0,0)$.

Build the descending subchain of $\left\{c_{1}, \ldots, c_{12}\right\}$ given by $d_{1}=(1,1), d_{2}=c_{3}=b_{2}=(1,1), d_{3}=$ $c_{6}=b_{3}=(0.5,1), d_{4}=c_{9}=b_{5}=(0,0), d_{5}=c_{12}=$ $b_{6}=(0,0)$.

Hence $\operatorname{orness}\left(F_{\alpha}\right)=b_{3}$.

If we consider another weighting vector $\beta=$ $((0.5,0),(0,0),(1,0),(0.5,1),(1,1)) \in L^{5}$, a similar calculation gives orness $\left(F_{\beta}\right)=b_{4}$, which means that $F_{\beta}$ is further away from the OR-operator than $F_{\alpha}$.

Proposition 12. In the conditions of Definition 9:

1. The descending chain $d_{1} \geq_{L} \cdots \geq_{L} d_{n}$ only depends on the number $n$ of weights.

2. If $\alpha=\left(1_{L}, 0_{L}, \ldots, 0_{L}\right) \in L^{n}$, then $\operatorname{orness}\left(F_{\alpha}\right)=d_{1}=1_{L}$.

3. If $\alpha=\left(0_{L}, \ldots, 0_{L}, 1_{L}\right) \in L^{n}$, then $\operatorname{orness}\left(F_{\alpha}\right)=d_{n}=0_{L}$.

4. If $\alpha \in L^{n}$ satisfies that $\alpha_{k}=1_{L}$ for some $1<k<n$ and $\alpha_{i}=0_{L}$ for any $i \neq k$, then $\operatorname{orness}\left(F_{\alpha}\right)=d_{k}=c_{(k-1) l}$, a component of the chain $b_{1} \geq_{L} \cdots \geq_{L} b_{l}$.

Obviously, Yager's orness for real-valued operators cannot be a particular case of Definition 9 because $[0,1]$ is not a finite lattice. However, it is possible to consider a finite sublattice of $[0,1]$ and compare the measure calculated as in Definition 9 with Yager's one, as has been done in the following proposition.

Proposition 13. Let $T$ be the t-norm defined on the real interval $[0,1]$ by the product and $S$ the $t$ conorm given by $S(a, b)=\min \{a+b, 1\}$. Given a distributive weighting vector $\alpha=\left(\alpha_{1}, \ldots, \alpha_{n}\right) \in$ $[0,1]^{n}$, consider the descending chain contained in 
$[0,1], L=\left\{a_{1}, \ldots, a_{n}\right\}$ where $a_{k}=\frac{n-k}{n-1}$ for every $1 \leq k \leq n$.

Then, the orness of the OWA operator $F_{\alpha}$ calculated on $L$ as in Definition 9 agrees with that given by Yager.

\section{Product lattices}

This section analyzes the orness of OWA operators defined on product lattices in order to apply them to a problem of color image processing.

Let $\left(L, \leq_{L}, T, S\right)$ be a finite lattice and consider, for any $r \in \mathbb{N}$ with $r>2$, the product lattice $\left(L^{r}, \leq_{L^{r}}, \mathbf{T}, \mathbf{S}\right)$ where

$$
L^{r}=\left\{\left(p_{1}, \ldots, p_{r}\right) \mid p_{i} \in L, 1 \leq i \leq r\right\}
$$

with the order relation $\leq_{L^{r}}$ given by: $\left(p_{1}, \ldots, p_{r}\right) \leq_{L^{r}}\left(q_{1}, \ldots, q_{r}\right)$ if and only if $p_{i} \leq_{L} q_{i}$ for all $1 \leq i \leq r$. In addition, $\mathbf{T}: L^{r} \times L^{r} \rightarrow L^{r}$ is the t-norm given by

$$
\mathbf{T}\left[\left(p_{1}, . ., p_{r}\right),\left(q_{1}, . ., q_{r}\right)\right]=\left(T\left(p_{1}, q_{1}\right), . ., T\left(p_{r}, q_{r}\right)\right)
$$

and $\mathbf{S}: L^{r} \times L^{r} \rightarrow L^{r}$ is the t-conorm given by

$$
\mathbf{S}\left[\left(p_{1}, . ., p_{r}\right),\left(q_{1}, . ., q_{r}\right)\right]=\left(S\left(p_{1}, q_{1}\right), . ., S\left(p_{r}, q_{r}\right)\right) .
$$

Theorem 3.4 in [6] proves that the maps $\mathbf{T}$ and $\mathbf{S}$ are indeed a t-norm and a t-conorm respectively.

Next we show that the orness of any OWA operator defined on $L^{r}$ is related to the orness of some OWA operators defined on $L$. We start to study the chains of elements necessary to calculate the orness in $L^{r}$.

Theorem 14. Let $L=\left\{a_{1}, \ldots, a_{l}\right\}$ be a finite lattice and consider the l-dimensional interval $\left[b_{l}, \ldots, b_{1}\right]$ as in Def. 9. For any $r \geq 2$, the $l^{r}$ dimensional interval $\left[\mathbf{v}_{l^{r}}, \ldots, \mathbf{v}_{1}\right]$ calculated in the same way for the lattice $L^{r}$ consists of the following elements:

$$
\begin{aligned}
& \mathbf{v}_{1}=\mathbf{v}_{2}=\cdots=\mathbf{v}_{l^{r-1}}=\left(b_{1}, \ldots, b_{1}\right) \in L^{r} \\
& \mathbf{v}_{l^{r-1}+1}=\cdots=\mathbf{v}_{2 l^{r-1}}=\left(b_{2}, \ldots, b_{2}\right) \in L^{r} \\
& \vdots \\
& \mathbf{v}_{(l-1) l^{r-1}+1}=\cdots=\mathbf{v}_{l^{r}}=\left(b_{l}, \ldots, b_{l}\right) \in L^{r}
\end{aligned}
$$

Corollary 15. In the conditions of Theorem 14, for any weighting vector on $\left(L^{r}, \leq_{L^{r}}, \mathbf{T}, \mathbf{S}\right), \alpha=$ $\left(\alpha_{1}, \ldots, \alpha_{n}\right)$, with $\alpha_{i}=\left(\alpha_{i 1}, \ldots, \alpha_{i r}\right)$ for any $1 \leq$ $i \leq n$, call $\beta_{j}=\left(\alpha_{1 j}, \ldots, \alpha_{n j}\right)$ for each $1 \leq j \leq r$. Then

1. $\operatorname{orness}_{L^{r}}\left(F_{\alpha}\right)=\left(\operatorname{orness}_{L}\left(F_{\beta_{1}}\right), . ., \operatorname{orness}_{L}\left(F_{\beta_{r}}\right)\right)$.

2. For any $n$-dimensional vector on $L^{r}$, $\left(\mathbf{a}_{1}, \ldots, \mathbf{a}_{n}\right)$ with $\mathbf{a}_{i}=\left(a_{i 1}, \ldots, a_{i r}\right) \in L^{r}$ for any $1 \leq i \leq n, F_{\alpha}\left(\mathbf{a}_{1}, \ldots, \mathbf{a}_{n}\right)=$ $\left(F_{\beta_{1}}\left(a_{11}, \ldots, a_{n 1}\right), \ldots, F_{\beta_{r}}\left(a_{1 r}, \ldots, a_{n r}\right)\right)$.

\section{Color image reduction using OWA operators defined on product lattices}

In this work we consider an image of $H \times W$ pixels in the RGB color scheme as a set of $H \times W$ elements arranged in rows and columns. Each element of a color image is represented by $q_{i, j}$ with $i \in\{1, \ldots, H\}, j \in\{1, \ldots, W\}$. Each element has associated three integer numbers between 0 and 255 that represent the level of red, green and blue color. If we call $L=\{0,1, \ldots, 255\}$, we can see that $q_{i, j} \in L^{3}$. Therefore, we establish a relation between the RGB color scheme and the product lattice given by $L \times L \times L$.

Given a color image $Q$ of $H \times W$ pixels and a reduction block size $n$, the reduction algorithm we propose consists in the following steps:

1. select an OWA operator defined on the lattice $L \times L \times L$

2. divide the image into non-overlapping blocks of $n \times n$ pixels. In this way, the reduced image $Q^{\prime}$ will have $\frac{H}{n} \times \frac{W}{n}$ pixels;

3 . for each pixel in the image $Q$, apply the considered lattice OWA operator to the pixels within the block;

4. rearrange the result of the OWA operator in the corresponding pixel of the reduced image.

Consider the image of $510 \times 510$ pixels shown in Figure 1. We are going to analyze the reduced images obtained taking $n=3$ as reduction block size and three different OWA operators defined by the minimum t-norm $\mathbf{T}_{M}$ and the maximum t-conorm $\mathbf{S}_{M}$. Each OWA operator is associated with the following weighting vectors:

$$
\begin{array}{cc}
\alpha^{1}=\quad & ((0,0,64),(0,64,64),(64,64,64), \\
& (0,0,128),(0,128,128),(128,128,128), \\
& (0,0,255),(0,255,255),(255,255,255)) \\
\alpha^{2}=\quad((0,0,128),(0,128,128),(128,128,128), & (0,0,64),(0,64,64),(64,64,64), \\
& (0,0,255),(0,255,255),(255,255,255) \\
\alpha^{3}=\quad((0,0,255),(0,255,255),(255,255,255), \\
\quad(0,0,64),(0,64,64),(64,64,64), \\
\quad(0,0,128),(0,128,128),(128,128,128)
\end{array}
$$

The reduced images obtained are shown in Figure 2. Image (a), (b) and (c) correspond to the weighting vector $\alpha^{1}, \alpha^{2}$ and $\alpha^{3}$ respectively. Notice that the brightness of the reduced images are different, going from the darkest (a) to the brightest (c). If we analyze the qualitative orness measure of each OWA operator, we see that orness $\left(\alpha^{1}\right) \leq$ orness $\left(\alpha^{2}\right) \leq$ orness $\left(\alpha^{3}\right)$. In Table 1 , we show the orness measure of each OWA operator as well as the mean intensity of each color channel of the reduced images. Notice that there exist a close relation between the orness and the mean intensity. 


\begin{tabular}{ccccc} 
& Orness & Mean R intensity & Mean G intensity & Mean B intensity \\
\hline$\alpha^{1}$ & $(96,128,128)$ & 68.67 & 68.65 & 59.14 \\
$\alpha^{2}$ & $(128,128,128)$ & 70.81 & 71.15 & 63.06 \\
$\alpha^{3}$ & $(192,224,255)$ & 76.47 & 76.86 & 66.87
\end{tabular}

Table 1: Orness measure and mean red, green and blue intensities of the reduced images using the three OWA operators taking $\alpha^{1}, \alpha^{2}$ and $\alpha^{3}$ as weighting vectors.

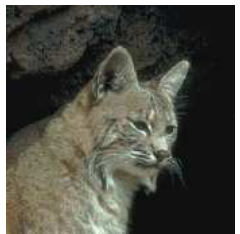

(a)

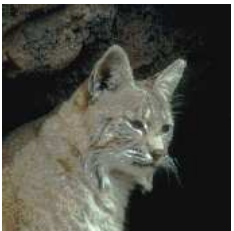

(b)

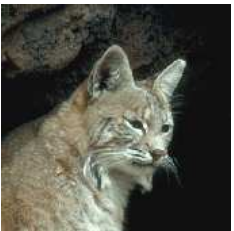

(c)

Figure 2: Reduced images obtained using the weighting vector $\alpha^{1}$ (a), $\alpha^{2}$ (b) and $\alpha^{3}$ (c).

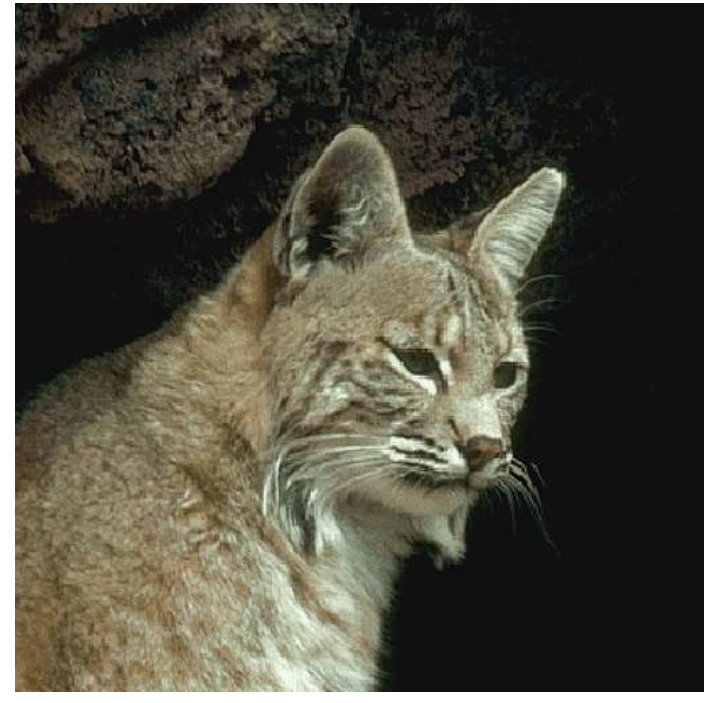

Figure 1: Original RGB image of $510 \times 510$ pixels.

\section{Conclusions}

In this work we have seen an application of OWA operators defined on a finite lattice for reducing color images in the RGB color scheme. We have seen that the intensities of the reduced images depend on the weighting vector considered.

In order to classify each OWA operator according to their proximity to an OR aggregation, we have made a preliminary study of a qualitative orness measure that behaves as the orness measure proposed by Yager for OWA operators defined on $[0,1]$.

In the application of image reduction, we have seen that the proposed orness measure give us information of the OWA operator considered. It also allows us to establish a close relation between the orness and the mean intensity of the images on each color channel.

\section{References}

[1] G. Beliakov, H. Bustince, D. Paternain, Image reduction using means on discrete product lattice, IEEE Transactions on Image Processing, 21: 1070-1083, 2012.

[2] B. De Baets, R. Mesiar, Triangular norms on product lattices, Fuzzy Sets and Systems, 104: $61-75,1999$.

[3] G. Grätzer, General lattice theory, Birkhäuser Verlag, Basel, 1978.

[4] M. Komorníková, R. Mesiar, Aggregation functions on bounded partially ordered sets and their classification, Fuzzy Sets and Systems, 175: 48 $-56,2011$.

[5] I. Lizasoain, C. Moreno, OWA operators defined on complete lattices, Fuzzy Sets and Systems 224: 36 - -52, 2013.

[6] I. Lizasoain, G. Ochoa, Generalized Atanassov's operators defined on lattice multisets, Information Sciences 278: 408 - - 422, 2014.

[7] D. Paternain, J. Fernandez, H. Bustince, R. Mesiar, G. Beliakov, Construction of image reduction operators using averaging aggregation functions, Fuzzy Sets and Systems 261: 87-111, 2015.

[8] R. R. Yager, On ordered weighting averaging aggregation operators in multicriteria decisionmaking, IEEE Transaction on Systems, Man and Cybernetics 18: 183 - 190, 1988.

[9] R. R. Yager, Families of OWA operators, Fuzzy Sets and Systems 59: 125 - -148, 1993. 